\title{
Über Patellarluxationen nach innen, zugleich ein Beitrag zur Therapie der Kniescheibenverrenkungen.
}

Von

\author{
Dr. Albrecht Mreyer, \\ Volontärassistent.
}

Mit 3 Abhildungen im Text.

(Eingegangen am 6. Dezember 1922.)

Karl hat im vergangenen Jahre (Arch. f. klin. Chirurg. Bd. 118) die bisher veröffentlichten Fälle von Patellarluxation zusammengestellt, er zählt 296 Verrenkungen. darunter nur 5 Luxationen nach innen. Schon aus diesem Zahlenverhältnis geht hervor, daß die Verrenkung der Kniescheibe nach innen etwas sehr Seltenes ist und diesem Umstande entnehme ich die Berechtigung dazu, vorliegenden Fall hier mitzuteilen. Ich lasse zunächst die Krankengeschichte folgen:

Frl. I. S., 21 Jahre alt, lief Ende Juli vergangenen Jahres durch weichen Sand und knickte dabe1 um, wobei sie plötzlich einen heftigen Schmerz im rechten Knie empfand und hinfiel. Nach einiger Zeit erhob sie sich wieder von selbst und ging ohne weitere Hilfe nach Hause. Sie hatte das Gefühl, als wenn sich etwas im Knir ausgehakt hätte, von selbst jedoch wieder sofort in Ordnung gekommen sei. Äußerlich hatte sie nichts bemerkt. Zu Hause machte sie einige Tage kühlende Limschlïge und fing dann allmählich wieder an zu laufen. 14 Tage später trat beim Baden nochmals plötzlich der heftige Schmerz auf, Patientin konnte jedoch sofort wieder gehen, ohne weitere Beschwerden zu haben. In der Folgezeit trat dasselbe Ereignis etwa alle drei bis vier Wochen ein, und zwar bei größeren Kraftanstrengungen, wie Laufen, Tanzen u. dgl.

In November $1921 \mathrm{kam}$ Frl. S. zum ersten Male in unsere Behandlung und es wurde eine starke Lockerung des Kniescheibenbandapparates festgestellt, welche sich darin äuBerte, daB die Patella sich weit auf den inneren Kondylus verschieben lie $B$, beim Loslassen jedoch sofort wieder in die normale Lage zurückkehrte.

Nach einer etwa dreiwöchigen Massagebehandlung des Oberschenkelstreckmuskels verschlimmerte sich das Leiden zunächst derart, daß die Verrenkung alle paar Tage auftrat, dann Besserung und Rückkehr zu den alten Zwischenrüumen und Eintreten der Luxation etwa alle drei bis vier Wochen. Mit der Zeit nahm die Häufigkeit des Auftretens jedoch wieder zu. Patientin, die wissenschaftliche Assistentin am hiesigen Pathologischen Institut ist, gewöhnte sich allmählich so daran, daß sie am Weitergehen durch die dabei eintretende Verrenkung nicht mehr gehindert wurde. Die Luxation trat nur bei heftig ausgeführter Streckung des Kniegelenkes auf und während sonst Aus- und Einrenkung unmittelbar aufeinanderfolgten, blieb in den letzten Wochen zweimal die Kniescheibe nach innen luxiert und sprang erst bei Beugung des Kniegelenkes in die normale Lage zurück. 
Aus der Vorgeschichte der Patientin bleibt noch nachzutragen, daß bei ihr eine angeborene linksseitige Hüftgelenkrerrenkung in Alter von $1 \frac{1}{2}$ Jahren in hiesiger Klinik unblutig eingerenkt worden ist. Seit AbschluB der damaligen Behandlung ist Frl. $S$. völlig beschwerdefrei. In Jahre 1912 erkrankte sie nach Knien in feuchtem Gras an einer Schleimbeutelentzïndung an beiden Kniegelenken. Es soll damals eine leichte Schwellung an beiden Kniegelenken vorhanden gewesen sein, welche nach einigen Tagen Puhe und Wickelung schnell zurückgegangen sei.

Aufnahme in die Klinik am 15. Mai 1922.

Untersuchungsbefund: Äußerlich ist am rechten Kniegelenk nichts Besonderes zu bemerken: Konturen im ganzen scharf, Umrisse der Kniescheibe an normaler Stelle, ein leichtes physiologisches X-Bein. Die Bewegliebkeit in beiden Kniegelenken ist völlig unbehindert. Bei Bougung ragt der imere Kondylus des Oberschenkels beiderseits stärker hervor als der äußere, or ist breiter und ron normaler Rundung. Bei gestreckten Unterschenkeln läßt sich die rechte Kniescheibe weit auf den Condylus internus versehieben, so dab sie sich ganz außerhalb des nornalen Kniescheibenbettes befindet. Nach außen dägegen ist ein Abdrängen nur um etwa $1 / 2 \mathrm{~cm}$ möglich. Die linke Kniescheibe ist nach heiden Seiten in abnormem Maße dislozierbar. Bei gebeugtem Knie ist die rechte Patella durch den Zug ihres Bandapparates bzw. des M. quadriceps fest und unverschieblich in ihrer Bahn zwischen den Kondylen fixiert. Die Muskulatur des rechten Oberschenkels zeigt starke Atrophie. $20 \mathrm{~cm}$ oberhalb des oberen Randes der Kniescheibe ergibt sich ein Unterschied im UmfangmaB von $3,0 \mathrm{~cm}$.

Operation am 17. Mai 1922 (Prof. Gocht) in Äthernarkose:

Hautschnitt au der Beugeseite, vom oberen Drittel des Oberschenkels bis unterhalb des rechten Fibulaköpfchens reichend. Die Sehne des langen Kopfes des M. biceps wird an ihrem Ansatz unter Mitnahme eines Streifens aus der Faszie der Mm. peronaei abgelöst, der Muskel innerhalb der ganzen Schnittlänge isoliert und dureh einen Tunnel im Fett zwischen Haut und Faszie zu einew schon vorher angelegten Längssehnitt über die Mitte der Patella hindurehgezogen. Der Hautschnitt auf der Beugeseite wird alsdann geschlossen, die Inzision auf der Streckseite bis auf die knöcherne Oberflïche der Patella und in die Quadrizepssehne und das Lig. patellae hinein vertieft. In diese Rinne wird die Bizepssehne hineingelagert, ihr Ende reicht bis zur Inzision im Lig. patellae; dann werden die periostalen und sehnigen Weichteile über ihr geschlossen.

Nach Raffung der Kapsel zum Vastus externus hin folgen Situationsnähte und schließlich die Hautnaht. Von einem Einriß der Kapsel an irgendeiner Stelle war nichts zu bemerken.

Der angelegte Schienenverband blieb einige Wochen liegen, dann wurde mit leichten Bewegungsversuchen begonnen. Eine Nachuntersuchung vier Monate nach der Operation ergibt völlige Beschwerdefreiheit.

Is handelt sich also um eine habituelle rechtsseitige Patellarluxation nach innen, welche hei heftig ausgeführten Streckbewegungen im Kniegelenk auftrat. Es findet sich außerdem bei der Patientin eine starke Lockerung des Bandapparates der linken Kniescheibe und in der Anamnese eine angeborene Verrenkung des linken Hüftgelenkes.

Bei der ersten Untersuchung in unserer Klinik schenkte man den doch immerhin ungenauen Angaben der an sich intelligenten Patientin zunächst wenig Glauben, da man angesichts der großen Seltenheit nicht dazu neigte, eine Juxation nach innen anzunehmen. Erst der objektive Befund, die starke passive Verschieblichkeit der rechten Kniescheibe nach der Innenseite zu und vor allem der weitere Verlauf führten zur Sicherstellung der Diagnose.

Es finden sich in der Literatur zahlreiche Veröffentlichungen über die Entstehungsweise der Verrenkungen und fast alle Autoren sind der Ansicht, daß das starke zahlenmäßige tberwiegen der Juxationen nach außen eine natürliche Folge der zugrunde liegenden anatomischen Verhältnisse sei, einige 
bezweifeln deshalb sogar überhaupt die Möglichkeit einer Luxation nach innen.

Bezüglich der Ätiologie unterscheidet man seit der Zeit, da die Kongenitalität eines Teiles der Luxationen feststeht:

1. angeborene,

2. traumatische,

3. pathologische Luxationen.

Während Malgaigne 1836 noch die bis dahin veröffentlichten Fialle von angeborenen Luxationen als solche ablehnte, fand Karl 1921 unter einer Gesamt. zahl von 296 Verrenkungen

137 kongenitale,

86 traumatische,

44 pathologische und

28 Fälle fraglichen Ursprunges.

Als Ursache für das Zustandekommen der kongenitalen Luxationen werden vor allem mechanische Einwirkungen im Uterus bzw. während der Geburt angeschuldigt, als da sind Verletzungen, Druckwirkungen, Erkrankungen des Fötus, Bändererschlaffung u. a. Auch ist behauptet worden, daß es sich um eine fehlerhafte Keimanlage der Patella hanclele.

Was die traumatischen Luxationen angeht, so ist hierbei zunächst zu unterscheiden $z$ wischen den Verrenkungen, welche infolge eines Traumas bei völlig normalen Verhältnissen entstehen, und Fällen, bei denen eine angeborene oder erworbene Disposition bereits bestand. Abgesehen von äußeren, direkten, scitlichen Gewalteinwirkungen spielen besonders Drehbewegungen im Kniegelenk (Gocht) und der. Muskelzug eine Rolle, und gerade durch das Zusammentreffen dieser beiden Momente kann es zu einem Zerreißen der Haltebänder und Herausspringen der Kniescheibe aus ihrem normalen Bett kommen. Es wird uns z. B. folgender Fall berichtet: Ein Patient will tine Maus totschlagen, wendet sich dabei plötzlich um und diese heftig ausgeführte Bewegung genügte, um die Kniescheibenverrenkung hervorzurufen.

Was im besonderen die Ursache für die Luxationen nach außen betrifft, so hat sich zuerst Streu bel (1866) näher mit dieser Frage befaßt. Er stellte Versuche an der lueiche an, es gelang ihm nicht eine Luxatio patellae nach innen herzustellen und er kam zu dem Schluß, daß für das Zustandekommen einer solchen eine weitgehende Veränderung des Bandapparates Vorbedingung sei und schon deshalb die Luxationen nach außen weitaus die Mehrzahl der seitlichen Verrenkungen darstellen müßten.

Von einer großen Anzahl von Autoren ist das physiologische Genu valgum als prädisponierendes Moment für die Verrenkungen nach außen angesehen worden, gegen welche Ansicht mehrere Statistiken sprechen, und zwar insofern, als sich in ihnen etwa die gleiche Anzahl von Männern wie Fraven an Patellarverrenkungen erkrankt findet, während das physiologische $X$-Bein normalerweise doch bei der Frau stärker ausgeprägt ist als beim Manne. Diesem gegenüber stehen die Erfahrungen Gochts, der etwa 30 Patellarverrenkungen, sämtlich bei Patienten weiblichen Geschlechts, beobachtete. Anatomische Untersuchungen haben gezeigt, daß die Patella näher am Condylus externus liegt und die laterale Gelenkfläche der Kniescheibe bei raschem Wechsel der 
Bengung und Streckung sich am äußeren Kondylus aufrichtet. Weiterhin hat besonders $H$. v. Meyer (1880) darauf hingewiesen, daß die Richtungsachse des II. quadriceps in ihrem Verlaufe vom Becken bis zur Tuberositas tibiae einen im Knie nach außen offenen stumpfen Winkel bildet. Es wird bei jeder Kontraktion dadurch ein gewisser Zug an der Patella nach außen ausgeübt, da sich dieser Winkel natürlich auszugleichen sucht.

Andere Autoren betonen vor allem das stärkere Hervorragen des inneren und die Abflachung des äußeren Kondylus, welche Erscheinung sich oft besonders ausgeprägt findet als Folge von Rachitis.

In vielen Veröffentlichungen ist auf die Einwirkung des rachitischen Genu valgum hingewiesen worden. Gegenüber der großen Häufigkeit desselben stellt die Patellarluxation ein sehr seltenes Leiden dar und daher erscheint es als minciestens gewngt, das $X$-Bein als primires, die Verrenkungen verursachendes Leiden anzusehen, wodurch nicht angezweifelt werden soll, daß es bei gewissen Fällen zur Entstehung der Luxationen sicherlich beiträgt.

Weiterhin ist behauptet worden, daß ein Versagen des Vastus medialis, welcher außer seiner Mitarbeit bei der Streckung des Unterschenkels normalerweise anch noch einen Zug auf den Streckapparat nach innen ausübt, die Ursache für das tberwiegen des Vastus lateralis und damit der Luxation nach außen sei.

In neverer Zeit wurde eine zu starke Außenrotation der unteren Femurepiphyse als Ursache angeschuldigt (H übscher u. a.), ferner wurde als mitwirkendes Moment die Schlußrotation im Kniegelenk genannt, welche bei den Verrenkungen nach außen in Betracht kommt, die bei Streckung des Kniegelenkes erfolgen. Daß überhaupt bei diesen Luxationen, welche bei forcierter Streckung entstehen, zum Teil ganz andere Momente mitspielen als bei denen. die bei Beugung des Kniegelenkes erfolgen, darauf ist vielfach hingewiesen worden und es wird weiter unten noch näher darauf eingegangen werden.

Nach Gesagtem drängt sich uns von selbst die Frage auf, wie ist das Zustandekommen der Verrenkung in vorliegendem Falle zu erblären und weshalb ist hier im Gegensatz zu der großen Anzahl von Luxationen nach außen die Verrenkung nach innen hin erfolgt?

Wie wir aus der Krankengeschichte ersehen, ist die Luxation in unserem Falle nur bei Streckung des Kniegelenkes erfolgt. Bei dieser Beinstellung befindet sich die untere Hälfte der Gelenkfläche der Patella auf dem oberen Teil der Fossa intercondylica. Unsere Patientin wiederholte uns nun immer wieder, daß gerade bei einer heftigen Streckbewegung die Luxation zustande käme. Während einer forcierten Kontraktion des MI. quadriceps befindet sich aber die Kniescheibe noch in einer weit höheren Stellung als eben erwähnt. Die Patella erreicht erst ihre höchste Stellung im Augenblick der stärksten Kontraktion des gesamten Muskelapparates. In diesem Moment nämlich berührt nur noch ein ganz schmaler Saum ihrer Gelenkflache die vorderen obersten Teile beider Kondylenanschwellungen. Sie befindet sich dabei fast völlig in der Fossa supratrochlearis. Man kann diese Bewegung der Patella bei ausgiebigster Anspannung des M. quadriceps leicht nachkontrollieren, wenn man sich mit gestreckten Knieen hinstellt und nun versucht, die Kniee weiter nach hinten durchzudrücken im Sinne des Genu recurvatum. Man beobachtet dann 
ganz deutlich, wie die Kniescheibe eine Bewegung nach oben macht und zugleich im ganzen sich etwas den Femurschaft nähert.

In diesem Moment des Maximums der Kontraktion des M. quadriceps wird gleichzcitig ein Zug auf die Patella nach außen ausgeübt, da die Achse des Streckmuskels mit dem Lig. patellae einen stumpfen Winkel bildet, dessen Scheitelpunkt in der Patella gelegen ist und dieser sich auszugleichen sucht. Tritt daher in diesem Augenblick ein Riß der Kapsel, etwa durch eine von innen her auf die Patella wirkende äußere Gewalt bei einem Trauma ein, so ist die Luxation nach außen die natürliche Folge der einwirkenden Kräfte. Aber auch eine Erschlaffung des Bandapparates, eine Drehung des Unterschenkels nach außen oder eine Abknickung des Unterschenkeis im Kniegelenk im Sinne des $\mathrm{X}$-Beines bei einer heftigen Bewegung genügen in manchen Fällen schon, um eine Verrenkung nach außen, ein Abgleiten der Patella auf die Vorderflache des äußeren Kondylus oder auf seine Außenseite zustande kommen zu lassen.

In unserem Falle mun ist nach dem objektiven Befunde eine Verschiebung der rechten Kniescheibe nach innen in einem die Norm weit übersteigenden Maße möglich: nach außen läßt sie sich dagegen nur ganz wenig aus ihrer Ruhelage passiv bewegen; mit anderen Worten der Bandapparat an der Innenseite besitzt die übliche Spannung, während außen anormale Verhältnisse vorliegen. In dem Augenblick der stärksten Muskelkontraktion verhindert also hier die normale Spannung der medialen Kapselteile die Luxation nach außen, während der äußere, schlaffere Bandapparat eine solche nach innen zuläßt. Wird nun bei maximal gespannter Muskulatur eine Drehbewegung im Knjegelenk im Sinne der Innenrotation des Unterschenkels bzw. bei feststehendem Fuß eine Außenrotation des Oberschenkels ausgeführt, so ist das Abweichen der Patella auf den inneren Kondylus die natürliche Folge. Die physiologisch einwirkenden Kräfte, die einen Zug auf die Patella in lateraler Richtung ausüben, werclen nach Entspannung des Streckmuskels und Beendigung der Drehbewegung augenblicklich ein Zurückkehren der Kniescheibe in ihre normale Lage be. wirken. Es kommt daher, wie auch die Patientin berichtet, zunächst nur zu einem momentanen Ausschnappen und erst nach Ausfahren des einmal betretenen Weges, nach einer woiteren Bänderdehnung. ereignet es sich, daß die Patella allf dem inneren Kondylus so lange bleibt, bis daß sie bei leichter Beugung des Kniegelenkes und damit verbundenem stärkeren Hervortreten des inneren Kondylus von selbst in die Trochlea zurückkehrt. Daß der Muskelapparat auch in unserem Falle bei Entstehung der Verrenkung eine gewisse, wenn auch nicht ursächliche Rolle (s. o.) spielt, beweist uns die oben berichtete vermehrte Häufigkeit des Auftretens der Luxation nach Massage der Oberschenkelstreckmuskulatur.

Die Einwirkungen der Drehbewegungen im Kniegelenk auf die Lage der Patella wird uns durch die Abbildungen $1-3$ veranschaulicht. Sie sind nach dem Skelett gezeichnet, das Lig. patellae und der M. rectus femoris sind durch ein Band schematisch dargestellt. Abb. 2 gibt die normalen Verhältnisse wieder. Abb. 1 zeigt das Abweichen der Patella auf den äußeren Kondylus bei Innendrehung des Oberschenkels gegen den feststehenden Unterschenkel, Abb. 3 die Verschiebung nach innen bei Außenrotation des Oberschenkels. Daß mindestens eine weitgehende Disposition zu den Luxationen durch die 
Rotationen geschaffen wird, erscheint hiernach als sicher. Ist nun außerde'm noch irgendein weiteres begünstigendes Moment vorhanden, so ist die Luxation unausbleiblich.

Eine weitere Frage ist die. woher stammt die konstatierte Erschlaffung des Gelenkbandapparates in unserem Falle? Aus der Krankengeschichte geht hervor, daß sich eine solche auch am linken Knie der Patientin findet. Hier ist eine ïbergroße Verschicblichkeit der Patella nach beiden Seiten hin vorhanden. Es läßt sich schwer entscheiden, ob diese beiderseitige Lockerung der Bänder eine Folge des angeblich vor einigen Jahren erlittenen Unfalles ist. Es erscheint unwahrscheinlich, da die damals konstatierte Schwellung der Kniegelenke angeblich schnell zurückgegangen ist und von einem Erguß nicht die Rede gewesen sein soll. Mit Rücksicht auf obige Ausführungen wäre noch zu sagen, daß wahrscheinlich die hier am linken Knie vorhandene beiderseitige Kapselerschlaffung der Grund dafür ist, daß hier keine Patellarluxation entstanden ist. Es besteht eben hier trotz der Erschlaffung ein gewisses Gleichgewicht in dem Spiel der bei stärkster Muskelkontraktion auf die Patella einwirkenden Kräfte, was rechts nicht der Fall ist.

Weitaus näher als ein Zusammenhang mit dem schon einige Jahre zurückliegenden Trauma erscheint mir die Annahme einer angebornen Anlage, auf welche Zusammenhänge Gocht in seiner Arbeit besonders aufmerksam macht und wofür

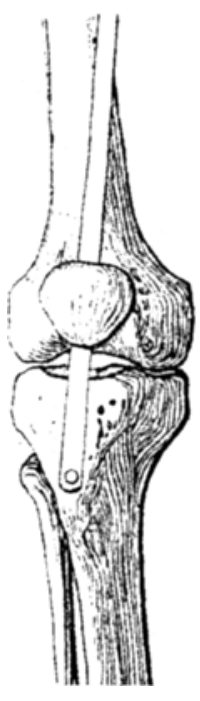

Abb. 1 .

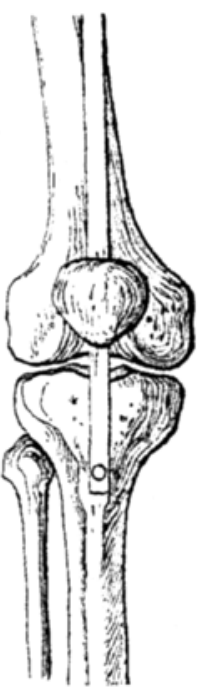

Abb. 2.

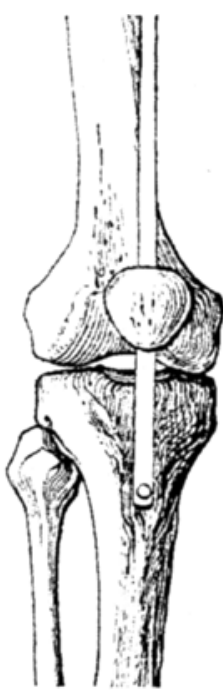

Abb. 3 . hier die früher vorhanden gewesene linksseitige angeborene Hüftverrenkung spricht. Bei der Durchsicht der einschlägigen Literatur habe ich vier Fülle ron kongenitaler Hüftgelenkluxation kombiniert mit Patellarluxationen gefunden (Bade, Bradford, Friedländer, Waas). Daß die angeborene Verrenkung des Hüftgelenkes vielfach mit anderen Mißbildungen vereint vorkommt, ist ja an sich eine bekannte Tatsache. Außerdem weist Wolff in seiner Veroffentlichung: ther einen Fall von angeborener ,willkürlicher" präfemoraler Kniegelenkslusation nebst anderweitigen angeborenen Anomalien fast sämtlicher Gelenke des Körpers auf die vielfach beobachtete multiple, angeborene Banderschlaffung hin. Es erscheint also naheliegend, daß es sich in unserem Falle um eine angeborene Hüftluxation, kombiniert nit beiderseitiger abnormer Schlaffheit des Bandapparates der Patella handelt, und daß auf Grund dieser angeborenen Disposition sich dann später anläßlich eines an sich bedeutungslosen Traumas, eines Fehltrittes, eine habituelle Patellarluxation entwickelt hat. 
Bei der Durchsicht der Literatur fand ich noch zehn Fälle von Luxationen nach innen, deren Krankengeschichten ich hier in Auszug folgen lassen will. Zum Teil waren mir nur ganz kurze Angaben zugänglich:

I. Key, Case of dislocation of the patella inwards. Lancet 1827 .

Eine etwa 30jährige Frau fiel mehrere Fuß herunter und stieß dabei mit dem rechten Knie gegen eine Mauer. Sie empfand sofort einen heftigen Schmerz und konute nicht mehr gehen. Reposition der Patella im Krankenhause. Exitus nach et wa vier Wochen an allgemeiner Sepsis, ausgehend ron dem verletzten Kniegelenk. Die Sektion ergilst einen RiB in der Sehne des Vastus externus und der Kapsel ober- und außerhalb der Kniescheibe. Fs wird ausdrücklich betont, $\mathrm{daB}$ dies ein wirklicher $\mathrm{RiB}$ war und keine Schädigung, die man der Entzïndung und ihren Folgen zuschreiben könnte.

II. Ha bgood, British ned. Journ. 1845.

Notiz äber einen von ihm beobachteten Fall von Luxation der Kniescheibe nach innen, welche, wie Habgood meint, wahrscheinlich durch Muskelzug entstanden sei.

III. Walther, erwähnt ron Malgaigne in der Gaz. méd. de Paris 1836.

Fall von Luxation der Patella nach innen bei einer 40jährigen Frau, ,aufgetreten nach einer Verrenkung in jugendlichem Alter". „Die hintere Fläche der Kniescheibe trat in Artikulation mit dem inneren Kondylus des Femur. Der äußere Kondylus war nicht glatt, sondern rauh."

IV. Putégnat, Journ. de chirurg. 1843.

Berichtet über einen Fall einer bilateralen habituellen Luxation nach innen nach Fall auf die Kniee bei einem zwölfjährigen Mädchen:

„Die Verrenkungen treten jetzt, fünf Jahre nach dem Unfall, so leicht ein. daß das junge Mädchen sich damit die Zeit vertreibt, mehr als hundertmal in einer Stunde die Kniescheiben aus- und einzurenken. Die Verrenkungen nach innen sind so vollstïndig, daß bei einander genäherten Oberschenkeln die vorderen Flächen der Kiniescheiben parallel stehen und genau aufeinander passen. Ich muB noch hinzufügen, $d_{a} B$ die rechte Kniescheibe sich leichter nach außen verrenkt, daß die linke sich leichter nach innen verrenkt, und $\mathrm{da} B$ alle beide sich dabei nicht umstïlpen. Die alleinige Kontraktion der Beinextensoren vermag die Knie nicht völlig zu strecken."

V. Bacon, A case of fracture and one of dislocation of the patella. Australasian Med. Gaz. Sydney 1843.

Eine Dame hatte beim Blindekuhspiel sich niedergehockt und dabei plötzlich eine Bewegung zur Seite gemacht, als sie merkte, daß sich irgend etwas ausgehakt hatte. Sie fiel dann ohne besondere $\mathrm{Wucht}$ hin und $\mathrm{Bac}$ on erhob bei seiner Untersuchung folgenden Befund:

Die Patella war nach innen verschoben, ihr oberer und äußerer Rand befand sich auf dem inneren Kondylus des Femur, ihre nach der Haut zu gelegene Fläche war dem anderen Oberschenkel zugewandt. Das Bein stand steif in leichter Beugestellung. Bacon renkte im Ätherrausch mit der Hand die Kniescheibe wieder ein.

VI. Putégnat, Journ. de chirurg. 1843.

Ein junges Mädchen verrenkte sich unwillkürlich eine Kniescheibe als sie mit ausgestreckten und aufgestützten Beinen am Tisch saß und ein Bein an das andere heranbringen wollte. Dasselbe war ihr schon einmal bei einem Abendessen passiert. Besonders bemerkenswert war, daß die ganz nach innen luxierte Kniescheibe so umgedreht war, daß ihre Gelenkfläche zur vorderen Fläche geworden war.

VII. Bartels, erwähnt bei Friedländer: Die habituelle Luxation der Patella. Inaug.-Diss. Berlin 1901.

Ein Fall von kompletter pathologischer habitueller Luxation nach innen bei einem. 17jührigen jungen Manne, dem sog. "Kautschukmann".

VIII. Périat: Luxation spontanée des deux rotules par relâchement originel des ligaments femororotuliens. Journ. de chirurg. de Malgaigne. 1845.

Anmerkung: Fall I-VI erwähnt bei Lonis Chevrier. Inaug-Diss. Paris 1904. Des lusations traumatiques de la rotule. 
Fall von kompletter intermittierender beiderseitiger Luxation nach innen bei einem $18 j$ juhrigen jungen Manne. Lig. patellae schlaff bei gut ausgebildeten Kondylen. Schlechter Gang beim Treppensteigen. Allgemeine Bänderschlaffheit.

IX. Smith: Dubliner pathol. Gesellschaft, zitiert nach Zielewicz. Berl. klin. Wochenschr. 1869 .

Fall von beiderseitiger, kompletter, permanenter, angeborener Luxation der Patella nach innen bei einem. Tungen von elf Jahren, zugleich fand sich hier eine beiderseitige Luxation des Kniegelenkes. Der M. quadriceps ist an beiden Beinen sehr schlaff und atrophisch, Lig. patellae schlaff, Kondylen normal, Gang gut. Med. 1876 .

$X$. Adelmann: Luxation des Knies nach außen und innen. Zeitschr. f. d. ges.

Fall von kompletter, permanenter, beiderseitiger, angeborener Luxation der Patella nach innen. Zugleich Verrenkung des Kniegelenkes nach außen, Atrophie des Vastus internus, Rectus femoris nach innen gezogen, Condylus internus sehr klein, Condylus externns sehr groß.

Fall I, III und IV stellen offenbar Verrenkungen der Kniescheibe rein traumatischen Ursprunges dar.

Bei Fall I handelt es sich um einen heftigen Stoß mit dem Knie gegen eine Mauer bei einem Sturz, bei Fall III um eine in jugendlichem Alter erlittene Kniegelenksverrenkung und bei Fall IV um einen Fall auf beide Knie.

Im Gegensatz hierzu stehen die Luxationen II, V und VI, die auch infolge eines Traumas entstanden sind, bei denen wir jedoch nach obigen Ausfïhrungen eine schon vorher vorhandene Disposition annehmen müssen. Während bei den erstgenannten Fällen rein traumatischen Ursprunges die Entstehungsweise sich aus der Art des Traumas von selbst ergibt, ist hier das Zustandekommen bei rlem an sich verhältnismäßig geringfügigen Trauma nur durch das Zusammentreffen von besonderen Umständen zu erklären.

Bei Fall II heißt es in der sehr kurzen Notiz nur: „,Probablement de cause musculaire". Habgood hat offenbar keine andere Erklärung finden können und leider fehlt jeder objektive Untersuchungsbefund.

Bei Fall $V$ ist die Luxation bei starker Beugung im Kniegelenk und Anspinnung der Muskulatur entstanden. Auch hier ist uns nicht mitgeteilt. ob etwa durch die anatomischen Verhältnisse eine gewisse Disposition zur Entstehung der Verrenkung gegeben war. Es fehlt auch jedwelche Angabe, ob eventuell eine Bänderzerreißung anzunehmen gewesen ist; das starke Hervor. treten des inneren Konlylus bei der Beugestellung erschwert zweifellos das Zustandekommen einer Verrenkung nach innen bei Flexionsstellung, welches andererseits begünstigt wird durch das gleichzeitige Fehlen des physiologischen Genu valgum.

Die Verrenkung VI hat bezüglich ihres Entstehungsmechanismus insofern Ähnlichkeit mit unserem Fall, als hier die Luxation in voller Streckstellung und in dem Moment stärkster Kontraktion der Streckmuskulatur entstanden ist. Wir müssen in diesem Fall zweifellos eine überwiegende Wirkung des Vastus internus neben einer gleichzeitig stattgefundenen Rotationsbewegung im Kniegelenk und einer gewissen, schon varher vorhanden gewesenen Lockerung des Bandapparates annehmen, wollen wir nicht sogar einen Einriß der

An merkung: Fall VIII $-\mathrm{X}$ sind erwähnt bei H. Bogen: Über familiäre Luxation und Kleinheit der Patella. Zeitschr. f. orthop. Chirurg. Bd. 16. 1902. 
von der Kniescheibe nach außen gelegenen Bänder durch Muskelzug konstruieren.

Die Luxationen VIII und $\mathrm{X}$ sind uns als kongenitalte gemeldet. Bei Fall IX und $\mathrm{X}$ ist die Patellarluxation verbunden mit einer angeborenen Verrenkung des Kniegelenkes. Bei Fall VIII handelt es sich offenbar um eine angeborene Anomalie des gesamten Bandapparates, von welcher Erseheinung schon oben einmal die Rede war.

Die von Friedläıder erwähnte Luxation VII, bei dem sog. Kautschukmann, entbehrt jeder näheren Angabe, die Originalarbeit war mir nicht zugänglich. Nur der Vollständigkeit halber habe ich die Notiz hier beigefügt.

Uber die bei diesen zehn Fällen von Patellarluxation nach innen angewandten Behandlungsmethoden wird uns bei einigen von ihnen berichtet, da $B$ die betreffenden Autoren die frisch entstandene Luxation unblutig manuell eingerenkt haben, was in keinem Falle größere Schwierigkeiten machte. Uber die Behandlung der habituell gewordenen Verrenkungen ist nirgends etwas gesagt. Bezüglich der im vorliegenden Falle eingeschlagenen Thera pie ist hereits oben Näheres mitgeteilt worden.

Patellarluxationen waren schon in Altertum bekannt und im Laufe der Zeit ist eine große Anzahl der verschiedenartigsten Behandlungsverfahren angegeben worden. Vor Einführung der aseptischen Operationen beherrschten die Bandagen und Apparate das Feld. Heute sind sie durch den ungefährlich gewordenen blutigen Eingriff fast völlig verdrängt. Von der einfachen Testudo Galens an bis herauf zum komplizierten Hülsenapparat nit einem Korkring zur Fixierung der Patella (Wely) hat man unzählige Vorrichtungen erdacht, um das Herausgleiten der Kniescheibe aus ihrer natürlichen Bahn zu verhindern.

Wie schon oben gesagt, gibt man heute ganz allgemein der blutigen Operation den Vorzug, d. h. nur bei der Behandlung der habituellen Luxation. Die frischen traumatischen Fälle werden selbstverständlich auch jetzt noch zunächst unblutig eingerenkt und dann abgewartet, ob Neigung zum Wiedereintritt der Verrenkung bzw. zum Habitucllwerden besteht. War aber die Luxation von jeher habituell oder ist sie es bereits geworden, so muß heute die blutige Opera. tion als die allein richtige Therapie angesehen werden. Die Bandagen und Apparate erreichen entweder ihr Ziel nicht in vollkommener Weise oder sie behindern die Gebrauchsfähigkeit des Gliedes derartig, daß ihnen schon deshalb die Operation vorzuziehen ist.

Es sind bisher etwa 50 verschiedene Operationsmethoden angegeluen worden. $\mathrm{Da} \beta$ immer neue Vorschläge gemacht worden sind, findet seinen Grund darin, daß einerseits in vielen Fällen der Erfolg der Operation nicht so befriedigte, da $B$ sich die Methode allgemeine Anerkennung erworben hat, andererseits sind die anatomischen Verhältnisse bei den einzelnen Verrenkungen verschieden und machen schon hierdurch oft die Anwendung einer besonderen, dem Fall angepaßten Operationsmethode erforderlich.

Hübscher hat im Jahre 1909 (Zeitschr. f. orthop. Chirurg. Bd. 24) die bis dahin bekannt gewordenen Operationsmethoden in übersichtlicher Weise zusammengestellt. Die dort gegebene Tabelle habe ich auf Grund der mir 
zugänglichen Literatur bis zur heutigen Zeit ergänzt, um so einen Gesantiiberblick über die bisher veröffentlichten Verfahren zu ermöglichen.

Ahnliche Maßnahmen wie bei den habituellen Luxationen sind bei der (Lauernden Verlagerung der Patella erforderlich. Ich möchte daher hier noch zwei Operationsmethorlen erwähnen, welche in den letzten Jahren für dieses Leiden angegeben worden sind. Es ist selbstverständlich, daß hier besondere, die anatomischen Eigent ïmlichkeiten beriicksichtigende Eingriffe erforderlich sind.

Erlacher empfiehlt den M. semimembranosus bzw. den M. gracilis zusammen mit dem M. sartorius a uf die Patella zu verlagern, da nn zu osteotomieren, die Kapsel zu raffen und schließlich den Tractus iliotibialis und den Vastus lateralis $\mathrm{zu}$ durchtrennen.

Gocht verlagert, wenn bei Abriß notwendig, den Ansatz des Lig. patellae nedialwärts und implantiert einen der inneren Kapselhälfte entnommenen Muskelkapsellappen in die laterale Hälfte der Kapsel und läßt außerulem noch an der Patella einen aktiven Muskelzug nach innen angreifen in Gestalt des auf die Kniescheibe aufgenähten M. semitendinosus.

Wie wir aus der Aufstellung ersehen, haben sich seit dem Jahre 1909 besonders die Operationsmethoden stark vermehrt, welche an den Muskeln, der Kapsel und den Bändern in der Hauptsache angreifen. Schon früher angegebene Methoden wurden modifiziert und mehrere ganz neue Operationen sind zur Veröffentlichung gelangt.

Bei der großen Auswahl von Operationsmethoden wird man nur dann die richtige treffen, wenn man sich in jedem einzelnen Falle einer habituellen Luxation den Entstehungsmechanismus bis in das Einzelne hlarmacht. Es ist eben zu entscheiden, ob eine etwa vorhandene Cenu valgum-Stellung in so hohem Maße bei dem Zustandekommen der Luxation mitwirkt, claß seine Korrektur durch Osteotomie notwendig erscheint, oder etwa eine Auswärtsrotation des unteren Femurendes oder eine Verlagerung der Tuberositas tibiae als ursächliches Moment angeschuldigt werden muB und damit die entsprechenden Fingriffe notwendig macht.

Gilt es nun, weiterhin das gestörte Kräftegleichgewicht im Streckapparat wiederherzustellen, sei es nun, daß die Muskeln selbst ungleich wirken, sei es, daß die veründerte Spannung und Weite der Kapsel das Primäre ist, so stehen uns in der Hauptsache zwei Verfahren zur Verfügung:

Wir können eine aktive Muskelkraft am Streckapparat in querer Rich. tung ansetzen oder den Strechapparat nach innen zu durch Schaffung eines Haltebandes fixieren.

Für letzteren Weg stehen uns die verschicdensten Methoden zur Verfügung, wir können uns mit einer einfachen Kapselraffung begnügen, wir können eine Kapselplastik hinzufügen, einen Teil des Lig. patellae am inneren Kondylus der Tibia anheften oder endlich ein neues Halteband aus frei transplantierter Faszie herstellen. Die Einsetzung einer nenen, in medialer Richtung wirkenden Muskelkraft kann ebenfalls durch mehrere Verfahren erreicht werden. Es heißt die Wahl des Angriffspunktes und der Richtung für die neue Kraft zu treffen und unter den uns zur Verfügung stehenden Muskeln zu entscheiden. 
Operationen bei habitueller

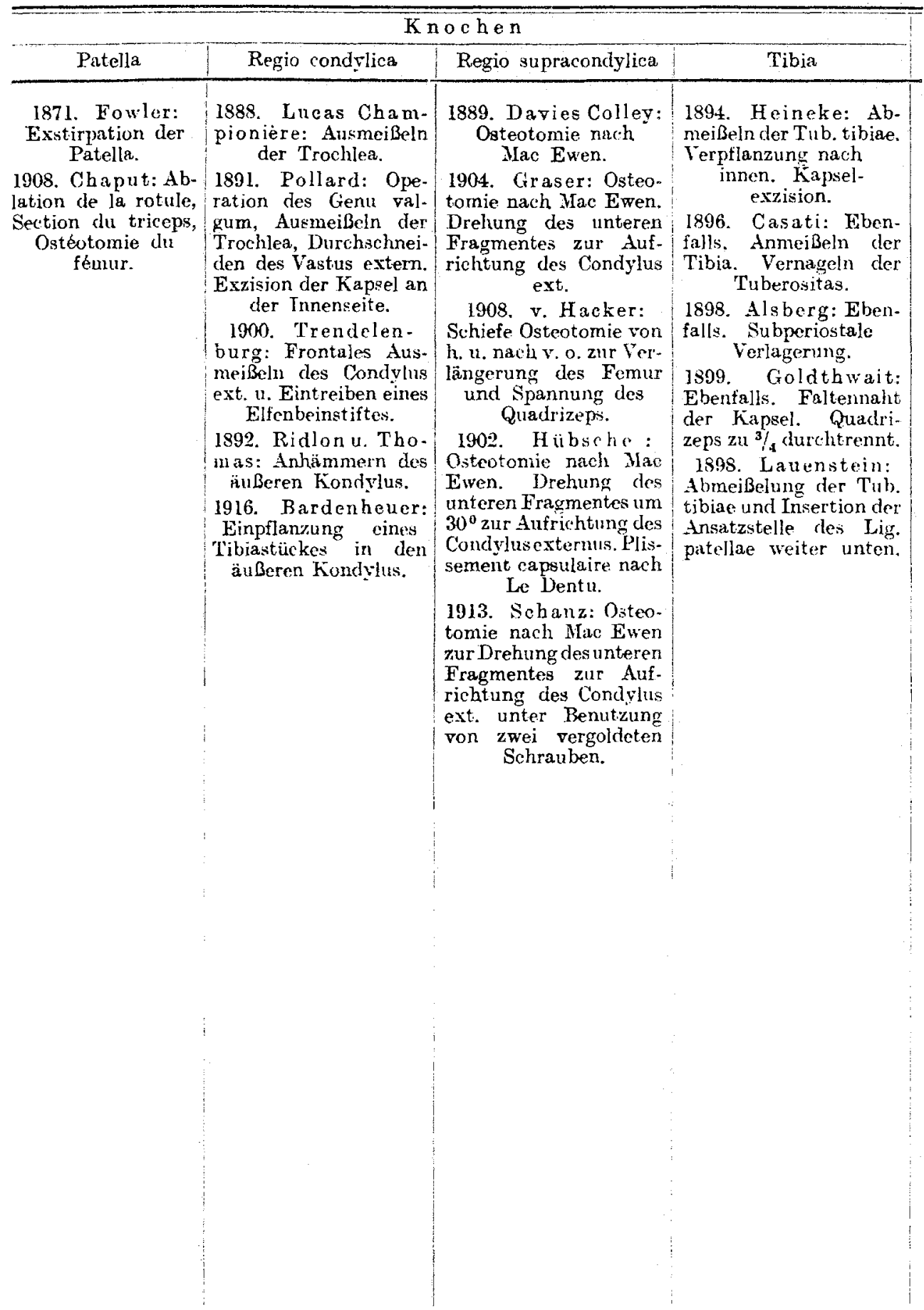


Luxation der Kniescheihe.

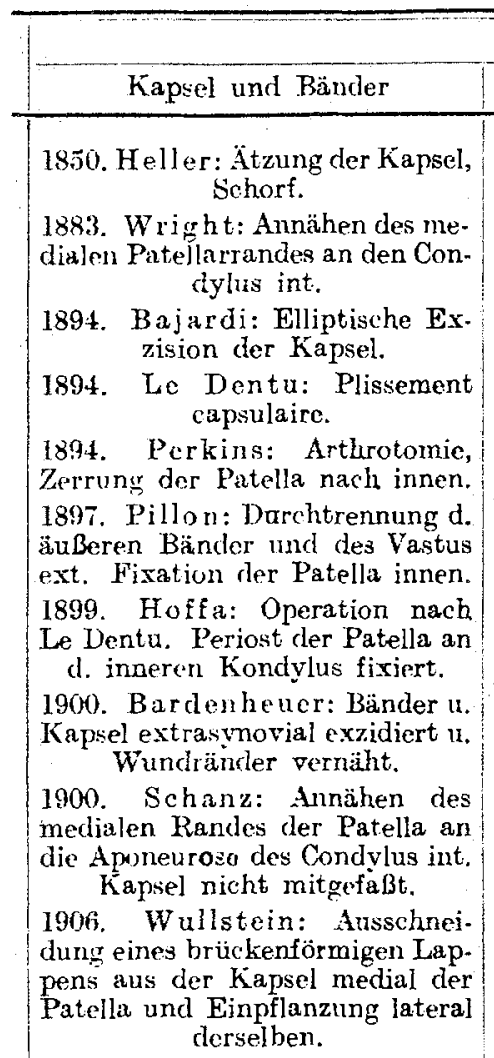

1917. Perthes: Raffung der Kapsel innen und Erweiterung derselben aıBen durch ein eingepflanztes Faszienstück.

1921. Ludloff: Durchtrennung des Tractus iliotibialis, der Kapsel auBen und der äußeren Partien des Vastus lat. Inzision in die Kapsel medial und Übereinander. nälien der Lefzen. Beugungsverband zur Delunung des Vastus lat. 1915. Hartleib: Fixiert den inneren Rand d. Patella durch einen frei transplantierten Fa:zienlappen am inneren Epikon. dylus. (Bei einer vertikalen Luxation.)

1917. Klapp: Fixation der $\mathrm{Pa}$. tella am Condylus med. femoris durch eine frei transplantierte Faszienschlinge.

Weichteile

Lig. patellae $\quad$ Muskeln

1884. Golding Bird: Tenotomie d. Lig. patellae. 1888. Roux: Verlagerung des Lig. patellae in ein exzidiertes Periostareieck am inneren Kondylus d. Tibia. Tenotomie des Vastus ext. Naht des Vastus internus. 1891. Heineke: Verpflan. zung des Lig. patellax nach innen. Fasziennaht zwischen Vastus int. und Sar. torius.

1904. Goldthwait: Spal. tung des Liys. patellize in zwci Hälften. AuBereHälfte abgelöst und unter der inneren durchgefuhrt an das Periont der Tibiainnen. fläche angenäht.

1907. Hübscher: Abspal. ten des inneren Drittels des Lig. patellae. Herunterziehen derPatella an diesem Zügel, Einnähen desselben unter eine Periostbrücke der medialen Tibiafläche. Dann Le Dentu.

1909. Dalla Vedova: Ab. spalten eines inneren Teiles des Lig. patellae. Einnähen desselben in horizontaler Richtung unter eine Periostbrïke des Planum epicond. int. des Femur. Lurchtrennung der äußeren Bänder und Kapse?.

\author{
(1)
}

1842. Guérin: Subkutane Tenutomie des Vastus ext. Rerressement des Genu valgum.

1902. Heasner: Semitendinosus möglichst in querer Richtung an die Quadrizenssehne grenäht.

1904. Lanz: Grazilis durch Faszienlücke des Vastus int. zum Innenrand der Patella. Seminiembranosus ebenfalls.

1904. Hof $f$ a: Semimenbranosus mit dem oberen Rand der Knic. scheibe vernäht.

1904. Ali Krogius: Längsschnitt am äußeren Rand der Patella mit Schonung der Synovialmembran. Brückenförmiger Lappen aus Vastus int. ind Kapsel. Einnähung dieses Iappens in den äuBeren Schnitt.

1914. Froelich: Ferlängerung des Quadrizeps in Z-Form. Dieser ist angeboren verkiurzt und ist Pepositionshindernis.

1911. Porzig: Operation von Goldthwait. Dazu Sartorius bzw. Semimembranosus + Semidentinosus auf dic Patella.

1914. Vulpius: Operation von Goldthwait, dann Osteotomie. Fixation des Sartorius an Patellainnenrand. Kajselraffung. 1919. Gocht: Verlarerung des Semidentinosus auf die Patella und Raffnähte in den Vastus med, an der Sehne des Rectus femoris beginnend.

1920. Dreyer: Bildung eines Zipfels aus dem Vastus fat. mit der Basis nach der Patclla. Nach Durchziehung unter der Sehne des Rectus femoris. Annähung des Zipfels am Vastus med. Durchtrennung der spannenden Zitge am Vastus lat. und Tractus iliotibialis. Kapselraffung innen. 1921. Drehmann: Fixierung des Rectus femoris nach medial durch eine Schlinge aus dem $\mathbf{M}$. gracilis. Grazilisansatz an den Condylus med. femoris verlegt. 1922. Gooht: Hab. Lixation nach innen: Verlagerung des $\mathrm{Y}$. biceps (Caput longum) auf die Patella und Raffung der Kapsel außen oben am Ansatz des Vastus externus. 
Die Verwendung der Kniebeugemuskeh hat den Forzug: daß durch sic erstens ein Halteband nach innen zu geschaffen wird und zweitens dieses Halteband kontraktil ist und daher stets unter Spannung steht, dem Zug des Vastus externus entgegenwirkend.

Bezüglich der speziell an unserer Klinik angewandten Operationsmethode verweise ich auf clie ausführliche Arbeit von Gocht: Uber die therpflanzung der Kniebeugenuskeln a uf den Kniestreckapparat (Arch. f. Orthop. u. Unfallheilk. Bd. 16. 1919). Auf Grund seiner langjährigen Erfahrungen tritt Gocht clort für die Verwendung des M. semitendinosus ein, welcher sich ganz besonders gut wegen seiner langen schmalen Sehne und seiner guten Zugrichtung für die Verlagerung auf die Patella eignet. Es werden außerdem noch Raffnähte in den sehnigen Teil des Vastuis medialis gelegt. In den eingehenden Ausführungen findet sich eine genaue Beschreibung des einfachen und doch das Ziel voll erreichenden Operationsverfahreas.

Analog dem Prinzip bei den Luxationen nach außen ist in vorliegendem Fall, wie oben berichtet, von Gocht der lange Kopf des M. biceps auf dic Patella verlagert worden, um dadurch einen Muskelzug auf die Kniescheibe in lateraler Richtung auszuüben und so ein erneutes Abgleiten derselben medial. wärts zu verhindern.

\section{Literatur.}

1. Bardenheuer s. Luxembourg: Med. Klinik. Nr. 24. 1914 - 2. Schanz s. Pauwels: Ref. Zeitschr. f. orthop. Chirurg. Bd. 31. 1913. - 3. Wullstein: 35. Kongr. d. dtsch. Ges. f. Chirurg. 1906. - 4. Perthes: Zentralbl. f. Chirurg. Nr. 12. 1917. -5. Ludloff: 0. K. XV. Ref. in Zeitschr. f. orthop. Chirurg. Bal. 40. - 6. Porzig: Zeitschr. f. orthop. Chirurg. Bd. 29. 1911. - 7. Vul pius: Med. Klinik. Nr. 6. 1914. 8. Gocht: Arth. f. Orthop. u. Unfullheilk. Bd. 16. - 9. Drcyer: Dtsch. med. Wochenschr. Nr. 18. 1920. - 10. D rehmann: Zentralbl. f. Chirurg. Nr. 18. 1921. 